\title{
Reactive oxygen species (ROS)-responsive nanoprobe for bioimaging and targeting therapy of osteoarthritis
}

Chong Shen ${ }^{1,2+}$, Ming Gao ${ }^{1,3+}$, Haimin Chen ${ }^{1 \dagger}$, Yanting Zhan ${ }^{1}$, Qiumei Lan ${ }^{1}$, Zhimin Li' ${ }^{1}$, Wei Xiong ${ }^{1}$, Zainen Qin ${ }^{1,3^{*}}$, Li Zheng ${ }^{1,3^{*}}$ and Jinmin Zhao ${ }^{1,3,4,5^{*}}$

\begin{abstract}
Stimulus-responsive therapy that allows precise imaging-guided therapy is limited for osteoarthritis (OA) therapy due to the selection of proper physiological markers as stimulus. Based on that the over-production of Reactive Oxygen Species (ROS) is associated with the progression in OA, we selected ROS as markers and designed a cartilage targeting and ROS-responsive theranostic nanoprobe that can be used for effective bioimaging and therapy of OA. This nanoprobe was fabricated by using PEG micelles modified with ROS-sensitive thioketal linkers (TK) and cartilage-targeting peptide, termed TKCP, which was then encapsulated with Dexamethasone (DEX) to form TKCP@DEX nanoparticles. Results showed that the nanoprobe can smartly "turn on" in response to excessive ROS and "turn off" in the normal joint. By applying different doses of ROS inducer and ROS inhibitor, this nanoprobe can emit ROS-dependent fluorescence according to the degree of OA severity, helpful to precise disease classification in clinic. Specifically targeting cartilage, TKCP@DEX could effectively respond to ROS and sustained release DEX to remarkably reduce cartilage damage in the OA joints. This smart, sensitive and endogenously activated ROS-responsive nanoprobe is promising for OA theranostics.
\end{abstract}

Keywords: ROS-responsive, Drug delivery, Targeting therapy, Osteoarthritis

\footnotetext{
*Correspondence: qn78495036@126.com; zhengli224@163.com; zhaojinmin@126.com

${ }^{\dagger}$ Chong Shen, Ming Gao and Haimin Chen contributed as first authors

${ }^{1}$ Guangxi Engineering Center in Biomedical Materials for Tissue

and Organ Regeneration, The First Affiliated Hospital of Guangxi Medical

University, Nanning 530021, China

Full list of author information is available at the end of the article
}

(c) The Author(s) 2021. Open Access This article is licensed under a Creative Commons Attribution 4.0 International License, which permits use, sharing, adaptation, distribution and reproduction in any medium or format, as long as you give appropriate credit to the original author(s) and the source, provide a link to the Creative Commons licence, and indicate if changes were made. The images or other third party material in this article are included in the article's Creative Commons licence, unless indicated otherwise in a credit line to the material. If material is not included in the article's Creative Commons licence and your intended use is not permitted by statutory regulation or exceeds the permitted use, you will need to obtain permission directly from the copyright holder. To view a copy of this licence, visit http://creativecommons.org/licenses/by/4.0/. The Creative Commons Public Domain Dedication waiver (http://creativeco mmons.org/publicdomain/zero/1.0/) applies to the data made available in this article, unless otherwise stated in a credit line to the data. 


\section{Graphical Abstract}

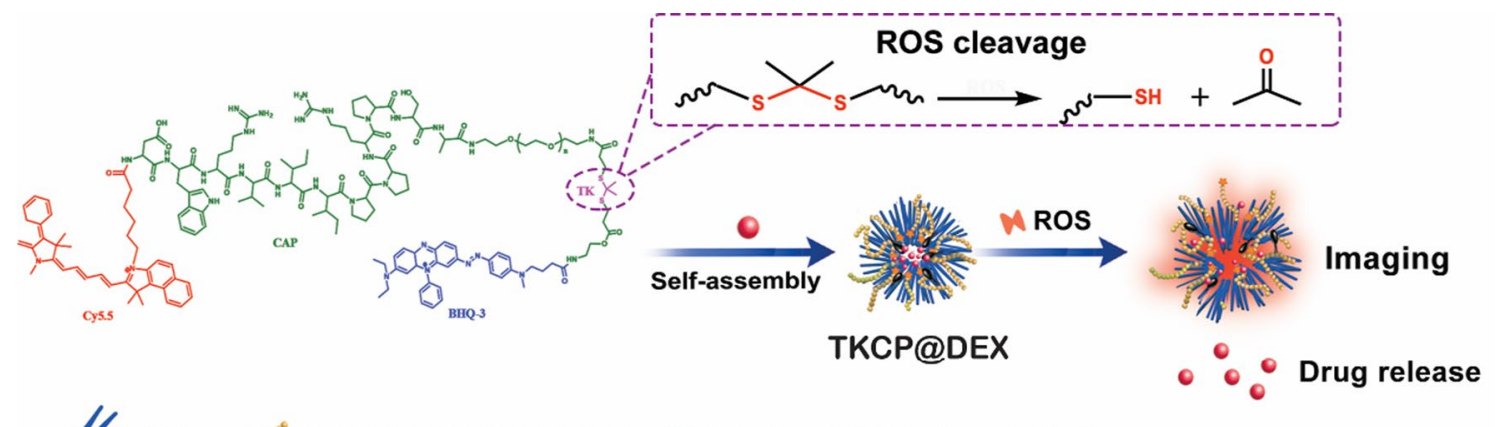

PEG CAPCDWRVIIPPRPSA $\sim$ Cy5.5 $\rho$ BHQ-3 $\odot$ DEX

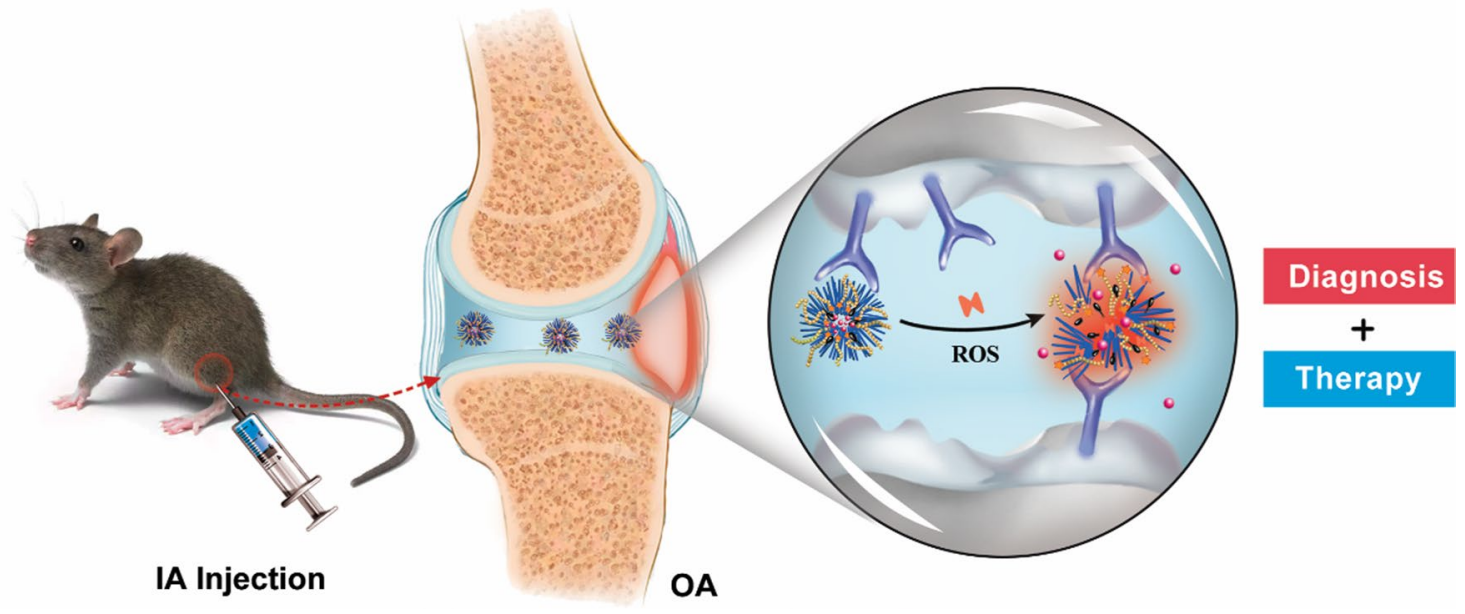

\section{Introduction}

Osteoarthritis (OA) is a chronic disease characterized by the progressive degeneration of cartilage that leads to joint pain and even serious disabilities of patients around the world $[1,2]$. At the end-stage of OA, the joints gradually lose function and need prosthetic replacements surgery [3]. Thus, it is necessary to highlight the diagnosis and treatment of OA.

Nowadays, endogenous-based fluorescent probes relying on certain physiological markers like NO [4] and MMP-13 [5-8] to generate fluorescence have been explored for detection of OA in vivo. However, these probes depending on pathological events are limited in clinical applications mainly due to the relatively low concentrations of endogenous biochemical markers [9-12], resulting in inaccurate and insensitive signals in the deep tissues. Moreover, most probes can only be used for monitoring and have little or no therapeutic effects on OA. Therefore, it is imperative to find remarkable physiological markers and design a responsive theranostic probe that can penetrate through the deep tissues and simultaneously have therapeutic potential for OA.
It is generally accepted that Reactive Oxygen Species (ROS) are important causative factors during the development of OA. It has been reported that the levels of ROS, e.g., $\mathrm{H}_{2} \mathrm{O}_{2}, \mathrm{O}_{2}^{-}, \mathrm{HO}^{-}$, and $\mathrm{HOCl}$, which maintain at a low level in normal articulatory [13], are dramatically increased (may up to $50-100 \mu \mathrm{M}$ ) in the joints of OA patients $[14,15]$. The over-generation of ROS elicits hyper-peroxidation, protein carbonylation and DNA damage, which has been considered as the main mechanism of cartilage cells loss and tissue damage [16]. But until now, a specific and biocompatible ROS-responsive system has not yet been moved forward to real-time monitoring and therapy of OA.

The drug delivery systems based on ROS-responsive functional moieties such as sulfide [17], phenylboronic acids and esters [18-20], selenium-containing linkage [21], peroxalate [22-24] etc., have been widely used for cancer treatment. Among various materials, nano-scaled ROS-responsive polyethylene glycol (PEG) polymers composed of thioketal linkers attracted most intention because of their sensitivity and responsiveness to endogenous ROS down to submicromolar concentrations 


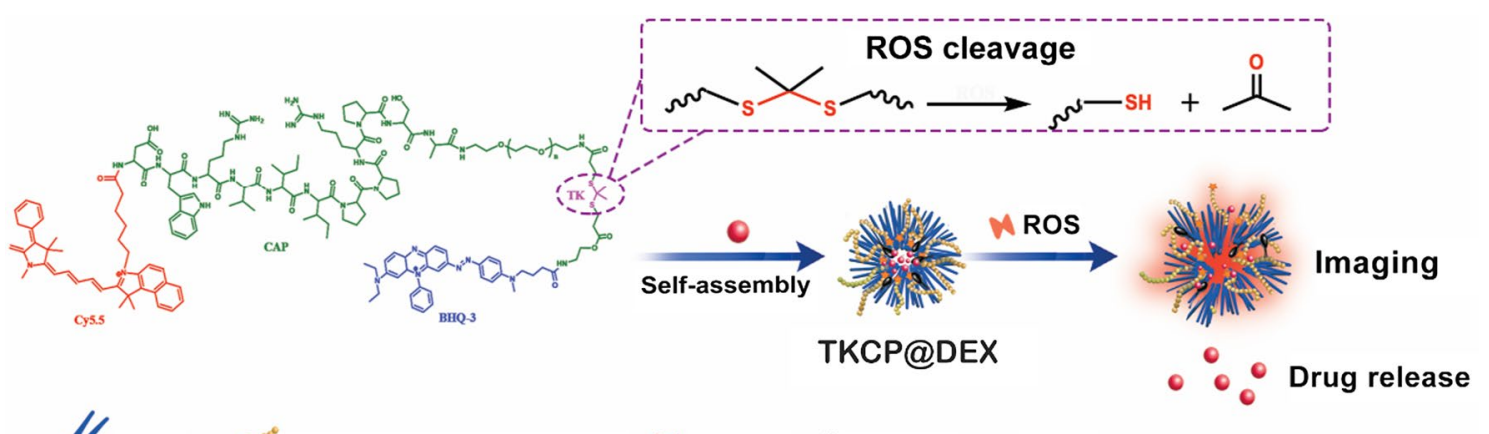

PEG CAPCDWRVIIPPRPSA $\$$ Cy5.5 $\rho$ BHQ-3

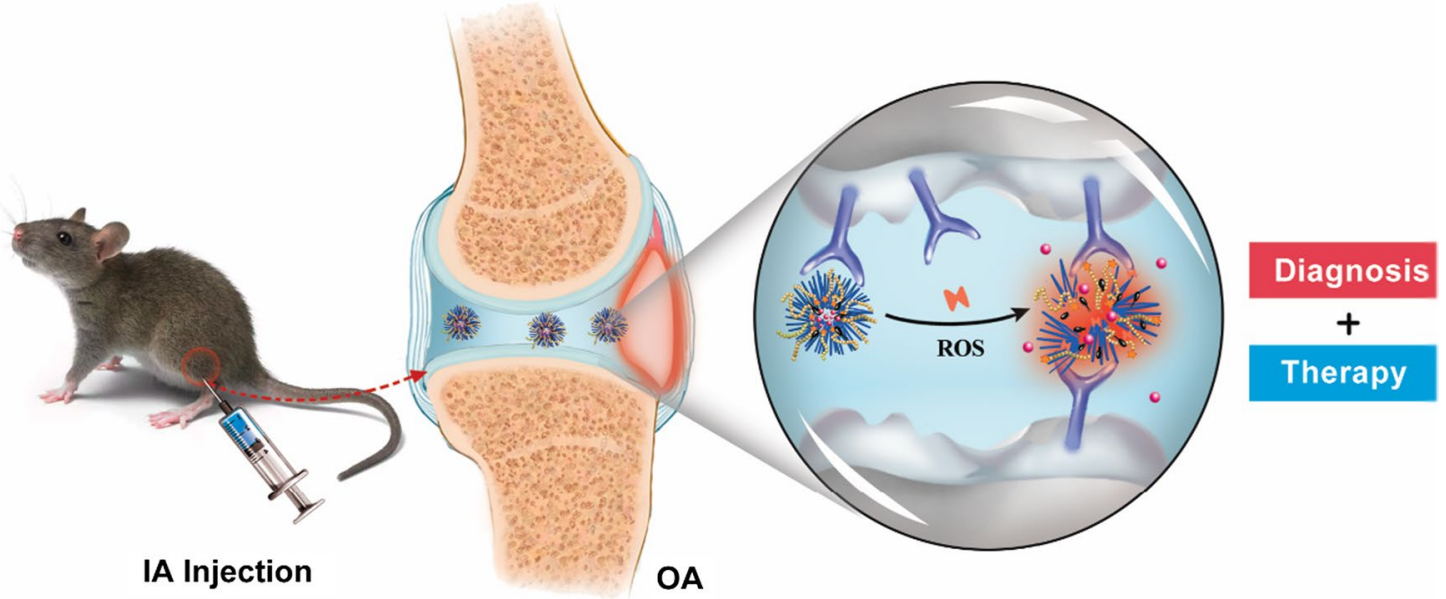

Scheme 1 Schematic illustration of the self-assembly of ROS-responsive nanoparticles for bioimaging and targeted therapy of osteoarthritis in vivo

$(\sim 100 \mu \mathrm{M})$ at deep physiological signaling levels [25-27]. PEG is usually employed to self-assemble micelles due to their excellent biocompatibility, which can shield from the mononuclear phagocyte system (MPS) and prevent host rejection after injected in vivo [28]. Moreover, the PEG micelles of sufficiently small size from 20 to $200 \mathrm{~nm}$ facilitates entry into the dense cartilage. Further modification with a targeted biomolecular ligand may resist rapid clearance from the joint site $[29,30]$. Thus, the ROS-responsive PEG micelles based on thioketal linkers may hold promise for OA treatment in clinical.

Herein, we developed a multifunctional ROS-activatable theranostic polymer nanoparticles that are capable of loading hydrophobic drug and self-reporting the payload release upon ROS stimulation. The nanoparticles are formed by the amphiphilic block copolymers consisting of Cy5.5 modified cartilage-targeting peptide (CAP, DWRVIIPPRPSA) [31, 32] and PEG modified an oxidation-responsive thioketal linkers (TK) hydrophobic block that contains Black Hole Quencher 3 (BHQ-3) as a quencher for Cy5.5, which was then encapsulated with Dexamethasone (DEX) to form TKCP@DEX nanoparticles. DEX is a broad-spectrum synthetic corticosteroid medicine with long-lasting anti-inflammatory effect to potently reduce glycosaminoglycan loss in OA-affected cartilage site [33-35]. As show in Scheme 1, the smart TKCP@DEX nanoparticles specifically target on articular cartilage by CAP and respond to the high level of ROS since the thioketal linkages were cleaved by abundant ROS in inflamed tissues, leading to gradual disassembly of the polymer to release Cy5.5 and drug. The increased distance between quencher (BHQ-3) and Cy5.5 enables stronger fluorescence signal from Cy5.5, providing effective monitoring of the progression of OA. But in normal condition where ROS are minimal, the fluorescence is turned off by BHQ-3. This smart cartilage-targeting ROS responsive theranostic nanoprobe is promising for $\mathrm{OA}$ therapy.

\section{Materials and methods}

Synthesis of ROS-responsive polymer (TKCP)

Here, thioketal were synthesized via their reaction with 3-mercaptopropionic acid and acetone. The fluorescent monomer modified polyethylene glycol (Cy5.5-CAPPEG) was synthesized following a route as shown in Additional file 1: Figs. S1 and S3. BHQ-3 was chosen as 
a quencher and co-polymerized with a ROS-cleavable thioketal-containing linker to prepare the Cy5.5-CAPPEG-TK-BHQ-3 (TKCP) (Additional file 1: Figs. S2, S3). For comparison, we also synthesized TKP without cartilage-targeting peptide (CAP) and CAPP without thioketal groups as controls (Additional file 1: Figs. S4, S5). The detailed preparation protocols of ROS-responsive polymers are presented in the Additional file 1 and verified by ${ }^{1} \mathrm{H}$ NMR spectra.

\section{DEX loading}

$20 \mathrm{mg}$ of nanoparticles was dispersed in $0.5 \mathrm{~mL}$ tetrahydrofuran (THF), followed by the addition of $10 \mathrm{mg}$ DEX (dissolved in $0.5 \mathrm{~mL}$ tetrahydrofuran). Then $10 \mathrm{~mL}$ deionized water with a syringe was slowly added in to the mixture. The mixture was moved to a dialysis bag ( $\mathrm{MWCO}=3 \mathrm{KDa}$, Sigma, USA) to dialyze with deionized water for $24 \mathrm{~h}$ to remove the unloaded drug. At last, the dried solid micelles were obtained by lyophilization. The drug loading and embedding ratio were measured by the High Performance Liquid Chromatography (HPLC) (Shimadzu, Japan), and then calculated by the following formula using:

$$
\begin{aligned}
\text { Drug Loading }(\%)= & \text { weight of DEX entrapped } \\
& / \text { weight of nanoparticles } \times 100 \%
\end{aligned}
$$

$$
\begin{aligned}
\text { Embedding ratio }(\%)= & \text { weight of DEX entrapped } \\
& / \text { weight of DEX feeding } \times 100 \%
\end{aligned}
$$

\section{Characterization}

The morphology of TKCP and TKCP@DEX was measured by transmission electron microscopy (TEM) (Bruker, Germany). The particle size distribution and zeta potential of the TKCP and TKCP@DEX were recorded using a dynamic light scattering (DLS) (Malvern, UK). The DLS was used to investigate the stability of TKCP incubated in PBS or in different concentrations of $\mathrm{KO}_{2}(0 \mu \mathrm{M}, 50 \mu \mathrm{M}$ and $100 \mu \mathrm{M})$ with or without ROS inhibitor (N-acetyl-L-cysteine, NAC) for 0,4 , $8,12,16,20$ and $24 \mathrm{~h}$. The Ultraviolet-visible (UV-VIS) absorbance spectra of Cy5.5, BHQ-3 and TKCP were detected by a microplate reader (Thermo Fisher Scientific, USA).

\section{Fluorescent recovery of TKCP}

The TKCP solution was added to different concentrations of $\mathrm{KO}_{2}(0 \mu \mathrm{M}, 50 \mu \mathrm{M}$ and $100 \mu \mathrm{M})$ with or without ROS inhibitor (N-acetyl-L-cysteine, NAC) and then incubated at $37^{\circ} \mathrm{C}$ for $0,1,2,4,8$ and $24 \mathrm{~h}$, respectively. The fluorescence signals were captured by using In-vivo Multispectral Imaging Systems (Bruker, Germany).

\section{DEX release study}

The DEX release profiles of TKCP@DEX NPs were determined by dialysis membrane method. Briefly, TKCP@ DEX NPs were placed in dialysis bags and immersed in $15 \mathrm{~mL}$ four different buffers: (1) PBS only; (2) PBS with $50 \mu \mathrm{M} \mathrm{KO}_{2}$; (3) PBS with $50 \mu \mathrm{M} \mathrm{KO}_{2}$ and its inhibitor; and (4) PBS with $100 \mu \mathrm{M} \mathrm{KO} \mathrm{KO}_{2} \mathrm{KO}_{2}$ was selected as a reagent to simulate the ROS microenvironment [25]. All solutions containing $1 \%$ tween 80 were shook constantly at $37^{\circ} \mathrm{C}$. At each time point, $1 \mathrm{~mL}$ of aliquots was removed from the release media and $1 \mathrm{~mL}$ of the same buffer was supplemented. The concentration of DEX release from TKCP@DEX NPs was measured by HPLC. The procedures were performed in triplicate.

\section{Chondrocytes isolation and culture}

Chondrocytes were isolated from the knee joints of 3-day-old C57BL6/J mice (the Animal Experimental Center of Guangxi Medical University, Nanning, China) by enzymatic digestion in aseptic conditions according to previous report [36]. Firstly, articular cartilages were digested in trypsin (Gibco, USA) for $40 \mathrm{~min}$ at $37^{\circ} \mathrm{C}$ and then minced and digested with $2 \mathrm{mg} / \mathrm{mL}$ collagenase II for $3 \mathrm{~h}$ at $37^{\circ} \mathrm{C}$. Secondly, the chondrocytes were centrifuged at $1000 \mathrm{rpm}$ for $5 \mathrm{~min}$ and then suspended in DMEM medium containing 10\% fetal bovine serum (FBS, Gibco, USA), 1\% penicillin and streptomycin (Solarbio, China). Then, they were transferred into a culture flask and cultured at $37{ }^{\circ} \mathrm{C}$ in a $5 \% \mathrm{CO}_{2}$-humidified incubator. The third generation of cells was collected for further experiments.

\section{In vitro cytotoxicity assay}

The cytotoxicity of TKCP and TKCP@DEX in chondrocytes was determined by Cell Counting Kit-8 (CCK-8, Japan). Briefly, cells were incubated in medium containing various concentrations of TKCP $(0,3,6,12,25,50$, $100,200 \mu \mathrm{g} / \mathrm{mL})$ or TKCP@DEX $(0,3,6,12,25,50,100$, $200 \mu \mathrm{g} / \mathrm{mL}$ ) for 24 and $48 \mathrm{~h}$. Afterward, each well was added with $10 \mu \mathrm{L}$ CCK- 8 and incubated for $4 \mathrm{~h}$ in humidified incubator. The absorbance of solutions was detected at a wavelength of $450 \mathrm{~nm}$ by a microplate reader (Thermo Fisher Scientific, USA). CCk-8 assay was also used to assess the cell viability of MIA-induced chondrocytes after treatment with DEX, CAPP@DEX (without ROS responsive linker, TK), TKP@DEX (without 
chondrocyte-affinity CAP peptide, CAP), or TKCP@ DEX for $24 \mathrm{~h}$.

\section{Hemolysis test}

The hemolysis ratio of TKCP at various concentrations $(50,100,200,400,800 \mu \mathrm{g} / \mathrm{mL})$ was performed in vitro [37]. The TKCP samples were dissolved in PBS at $37^{\circ} \mathrm{C}$. Then, $20.0 \mu \mathrm{L}$ of erythrocyte dispersion was added into the TKCP solution $(1.0 \mathrm{~mL})$ and the mixture was incubated for $1 \mathrm{~h}$ at $37{ }^{\circ} \mathrm{C}$. After centrifuged at $2000 \mathrm{rpm}$ for $10 \mathrm{~min}$, the hemoglobin in supernatant was measured using a microplate reader at $415 \mathrm{~nm}$. The positive and negative controls were determined by replacing the sample solution with ultrapure water and PBS, respectively. Experiments were performed for three times and the hemolysis rate (\%) was calculated using following equation:

$$
\left(A_{s}-A_{n}\right) /\left(A_{p}-A_{n}\right) \times 100
$$

where $A_{s}, A_{n}$, and $A_{p}$ mean absorbencies of the sample, negative control and positive control, respectively.

\section{Intracellular ROS detection and bioimaging}

We used monosodium iodoacetate (MIA), an inhibitor of glyceraldehyde-3-phosphate dehydrogenase activity, to induce oxidative stress injure and the pathological OA symptoms of chondrocytes [38]. Chondrocytes were seeded into 6-well or 24-well plates and divided into four groups: (1) control: chondrocytes cultured with medium only; (2) MIA $3 \mu \mathrm{M}$ : chondrocytes induced with $3 \mu \mathrm{M}$ MIA for $24 \mathrm{~h}$; (3) MIA + inhibitor: chondrocytes pretreated with $5 \mathrm{mM}$ NAC [39] (N-acetylcysteine, the antioxidant which can significantly prevent the production of ROS) for $1 \mathrm{~h}$ followed by addition with $3 \mu \mathrm{M}$ MIA for $24 \mathrm{~h}$; and (4) MIA $6 \mu \mathrm{M}$ : chondrocytes induced with $6 \mu \mathrm{M}$ MIA for $24 \mathrm{~h}$. Intracellular ROS production was determined by using a fluorescent 2,7-dichlorodihydrofluorescein diacetate (DCFH-DA) kit. Chondrocytes were harvested, and incubated with DCFH-DA $(10 \mu \mathrm{M})$ for $20 \mathrm{~min}$ in the dark at $37^{\circ} \mathrm{C}$. The chondrocytes were then washed three times with serum-free medium, and immediately detected by flow cytometer (BD, Biosciences, USA) [40].

In addition, cellular uptake and degradation of TKCP NPs induced by endogenous ROS were investigated. After treated with MIA, the chondrocytes were incubated with TKCP NPs for $4 \mathrm{~h}$, and then they were washed with PBS and fixed with $95 \%$ ethanol for $30 \mathrm{~min}$. Meanwhile, MIA $(3 \mu \mathrm{M})$ treated cells were also incubated with CAPP and TKP NPs as controls. Then the nuclei were counterstained with DAPI. Finally, the fluorescence images were photographed using a fluorescence inversion microscope (OLYMPUS, Japan).

\section{MIA-induced chondrocytes and treatment}

Chondrocytes were seeded into 24-well or 6-well plates and separated into five groups: (1) control: chondrocytes cultured with medium only; (2) MIA: chondrocytes induced with $3 \mu \mathrm{M}$ MIA; (3) MIA+DEX: chondrocytes pretreated with $3 \mu \mathrm{g} / \mathrm{mL}$ DEX for $1 \mathrm{~h}$ followed by addition with $3 \mu \mathrm{M}$ MIA for $24 \mathrm{~h}$; (4) MIA+TKP@DEX: chondrocytes pretreated with TKP@DEX (an equivalent DEX dose of $3 \mu \mathrm{g} / \mathrm{mL}$ ) for $1 \mathrm{~h}$ followed by addition with $3 \mu \mathrm{M}$ MIA for 24 h; (5) MIA + CAPP@DEX: chondrocytes pretreated with CAPP@DEX (an equivalent DEX dose of $3 \mu \mathrm{g} / \mathrm{mL}$ ) for $1 \mathrm{~h}$ followed by addition with $3 \mu \mathrm{M}$ MIA for $24 \mathrm{~h}$; and (6) MIA + TKCP@DEX: chondrocytes pretreated with TKCP@DEX (an equivalent DEX dose of $3 \mu \mathrm{g} / \mathrm{mL}$ ) for $1 \mathrm{~h}$ followed by addition with $3 \mu \mathrm{M}$ MIA for $24 \mathrm{~h}$.

\section{Quantitative real-time polymerase chain reaction (RT-qPCR) analysis}

The primer sequences for the OA-related genes are listed in Table 1. Total RNA was isolated using an RNA isolation kit (Tiangen Biotechnology, China). Then a reverse transcription kit (Takara, Japan) was used to reversely transcribe RNA to cDNA. Real-time PCR was conducted by a Light Cycle 96 system for $10 \mathrm{~min}$ at $95{ }^{\circ} \mathrm{C}, 15 \mathrm{~s}$ at $95{ }^{\circ} \mathrm{C}$, and $60 \mathrm{~s}$ at $60^{\circ} \mathrm{C}$. The relative gene expression levels were calculated using the $2^{-\Delta \Delta C T}$ method with $\beta$-actin as the control.

\section{Immunofluorescence}

The expression of OA catabolic biomarkers IL-6 and MMP-13 in chondrocytes was assessed by immunofluorescence. Chondrocytes were fixed with 95\% ethanol for $30 \mathrm{~min}$ and permeabilized with $0.1 \%$ Triton X-100 for $10 \mathrm{~min}$. Samples were incubated with primary antibody as follows: IL-6 (1:200, Boster, China), and MMP13 (1:200, Boster) at $4{ }^{\circ} \mathrm{C}$ overnight. Then the samples treated with the secondary antibodies FITC-anti-rabbit IgG (1:50, Boster) for $60 \mathrm{~min}$ at $37^{\circ} \mathrm{C}$ and counterstained with DAPI for $5 \mathrm{~min}$. Finally, the fluorescence images

Table 1 Primer sequences used in RT-qPCR experiments

\begin{tabular}{lll}
\hline mRNA & Forward primer & Reverse primer \\
\hline MMP-3 & CATCCCCTGATGTCCTCGTG & GATTTGCGCCAAAAGTGCCT \\
MMP-13 & TACCATCCTGCGACTCTTGC & TTCACCCACATCAGGCACTC \\
IL-6 & AGCCCACCAAGAACGATAGTC & GTGAAGTAGGGAAGGCCGTG \\
COL2A1 & ACACCGCTAACGTCCAGATG & TCGGTACTCGATGACGGTCT \\
$\beta$-actin & CCCATCTATGAGGGTTACGC & TTTAATGTCACGCACGATTTC
\end{tabular}


were photographed using a fluorescence inversion microscope (OLYMPUS, Japan).

\section{OA model and treatment}

All animal experiments were approved by the Ethics Committee of Guangxi Medical University. A total of 60 C57BL6/J (8 weeks old, male) were obtained for this experiment. To induce $\mathrm{OA}$, mice received a single IA injection of 0.05 or $0.1 \mathrm{mg}$ of MIA (Sigma, USA) after anesthesia [41-43]. After induction of OA model, the mice were randomly sorted into five groups $(n=6)$ : PBS group, IA injections of $50 \mu \mathrm{L}$ PBS; DEX group, IA injections of $50 \mu \mathrm{L}$ PBS with DEX $(1 \mathrm{mg} / \mathrm{kg})$; CAPP@DEX group, IA injections of $50 \mu \mathrm{L}$ PBS with CAPP@DEX (an equivalent DEX dose of $1 \mathrm{mg} / \mathrm{kg}$ ); TKP@DEX group, IA injections of $50 \mu \mathrm{L}$ PBS with TKP@DEX (an equivalent DEX dose of $1 \mathrm{mg} / \mathrm{kg}$ ) and TKCP@DEX group (an equivalent DEX dose of $1 \mathrm{mg} / \mathrm{kg}$ ). IA injections were performed twice a week. The mice in these groups were sacrificed for further analysis at 2 and 4 weeks after therapy.

\section{In vivo NIR bioimaging}

For in vivo bioimaging, mice were anesthetized by isoflurane. Each group of OA mice $(n=3)$ was IA injected with $50 \mu \mathrm{L}$ of $400 \mu \mathrm{g} / \mathrm{mL}$ TKCP, CAPP or TKP, and the normal mice $(n=3)$ were also IA injected with TKCP as control. The images were captured by an In-vivo Multispectral Imaging Systems (Bruker, Germany) at 0, 1, 2, 4, 7 and 14 days.

In addition, the fluorescence intensity of the TKCP was also investigated. The mice were randomly sorted into three groups $(n=3): 0.05$ mg MIA + inhibitor group, IA injection of $0.05 \mathrm{mg}$ MIA concomitant with $5 \mathrm{mM}$ NAC; $0.05 \mathrm{mg}$ MIA group, IA injection of $0.05 \mathrm{mg}$ MIA; $0.1 \mathrm{mg}$ MIA group, IA injection of $0.1 \mathrm{mg}$ MIA [44, 45]. Finally, the mice were sacrificed and the macroscopic evaluations of joints were performed.

\section{Macroscopic observation}

After 2 weeks or 4 weeks of treatment, the knee joints of mice were harvested for macroscopic evaluation according to the macroscopic scoring system (scale of $0-4$ ) by three independent observers [46].

\section{Histological analysis}

The joints were fixed in $4 \%$ paraformaldehyde and subsequently decalcified with a $14 \%$ ethylenediaminetetraacetic acid (EDTA) solution for ten days. Next the joints were embedded in paraffin and cut into $5 \mu \mathrm{m}$ thick slices by sharp blade. HE (Solarbio, China) and safranin $\mathrm{O}$-fast green (Solarbio, China) staining were performed for histomorphological analysis. The severities of OA were graded by three independent observers by using the
Osteoarthritis Research Society International (OARSI) score (scale of 0-24) [47]. Furthermore, Immunohistochemical staining for MMP13 (1:200, Boster) was performed to evaluate the anti-inflammatory effects of the probes.

\section{Statistical analysis}

All data are presented as the mean $\pm \mathrm{SD}$, and $\mathrm{p}<0.05$ was considered statistically significant. The significant differences among groups were evaluated by one-way ANOVA. Statistical analyses were conducted using SPSS statistics (SPSS 19.0).

\section{Results \\ Synthesis and fabrication of nanoreactors}

The successful synthesis of ROS responsive monomer and functional moiety were demonstrated by ${ }^{1} \mathrm{HNMR}$ spectra (Additional file 1: Figs. S1-S9). The transmission electron micrograph (TEM) showed that the amphiphilic polymer TKCP and TKCP@DEX could self-assemble into homogeneously spherical nanoparticles in aqueous solution (Fig. 1a and b). Furthermore, dynamic light scattering (DLS) analysis revealed that the average diameter of TKCP NPs was $\sim 60 \mathrm{~nm}$ (Fig. 1c). The zeta potential of TKCP NPs was close to neutral charges at $-0.13 \mathrm{mV}$ (Fig. 1d). After loading with DEX, the mean diameter of TKCP@DEX NPs increased up to 90 nm (Fig. 1b and c) and the zeta potential changed to $-0.43 \mathrm{mV}$ (Fig. 1d). The UV-vis spectrum also confirmed the successful construction of TKCP. As shown in Fig. 1e, characteristic peaks of Cy5.5 in Cy5.5-CAP-PEG-TK (TKCP) (red line) and $\mathrm{BHQ}-3$ (blue line) were demonstrated. There is a red shift induced by BHQ-3 in TKCP, which is synthesized by Cy5.5-CAP-PEG-TK (TKCP) and BHQ-3 through acyl chloride. To confirm the ROS-responsive fluorescence activation properties, different concentrations of $\mathrm{KO}_{2}$ were added to the TKCP NPs and their fluorescence intensity was monitored. In the absence of $\mathrm{KO}_{2}$, the fluorescence of TKCP was extremely weak over time due to the short distance between Cy5.5 and BHQ-3 (Fig. $1 \mathrm{f}$ and g). However, when $\mathrm{KO}_{2}(50 \mu \mathrm{M})$ was added, the TKCP showed gradual recovering of NIR fluorescence over incubation time due to that the ROS cleaved the thioketal of TKCP and increased the distance between Cy5.5 and BHQ-3. The recovery of fluorescence intensity was effectively accelerated with the increasing concentration of $\mathrm{KO}_{2}$ from 50 to $100 \mu \mathrm{M}$, but it was suppressed when an ROS inhibitor NAC was added together with the $\mathrm{KO}_{2}$. The TKCP NPs had good stability in PBS, showing no appreciable change in size distribution. On the contrary, the size rapidly increased under different concentration of $\mathrm{KO}_{2}(50 \mu \mathrm{M}$ and $100 \mu \mathrm{M})$ as the inducer of ROS, 

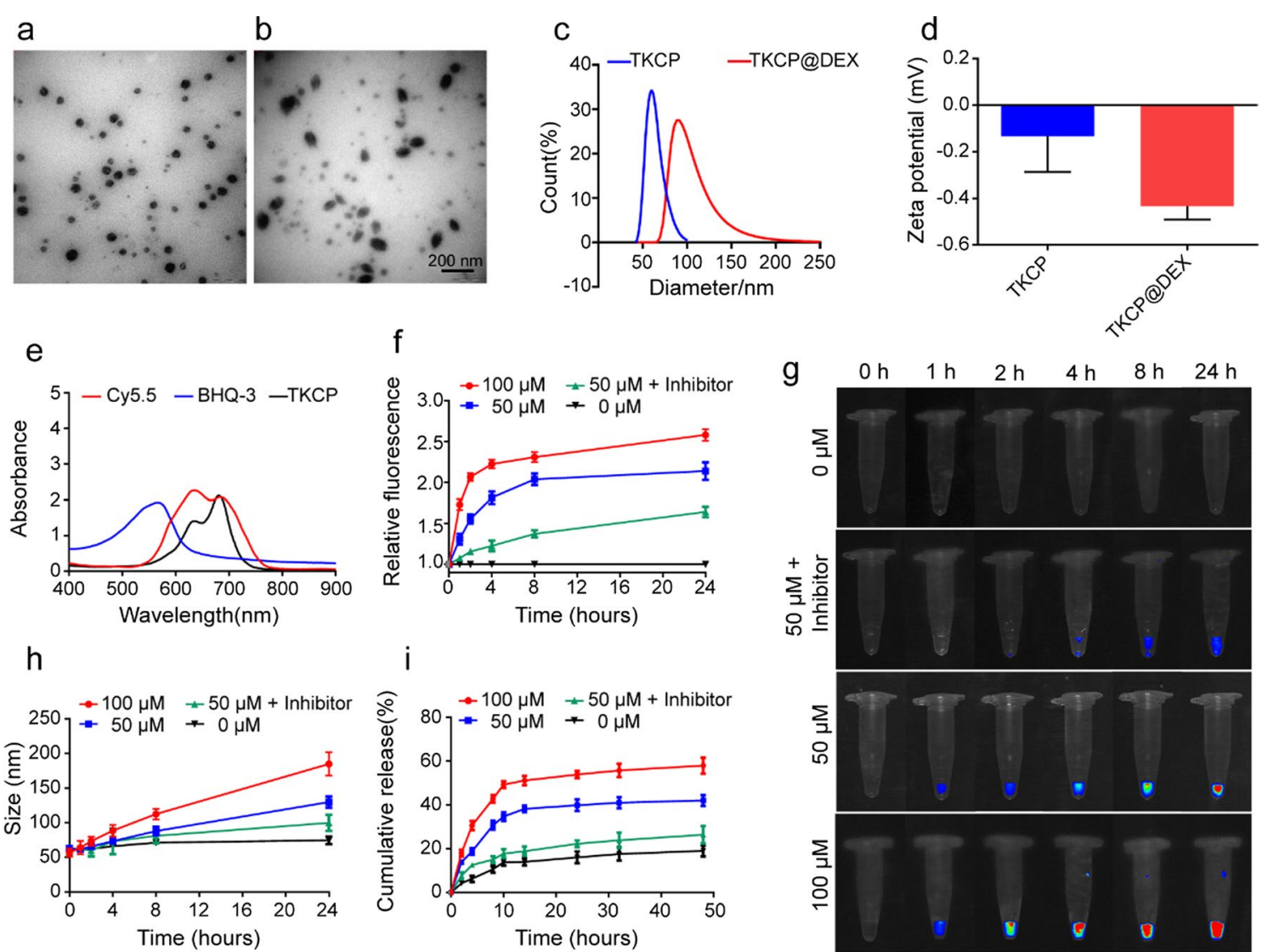

Fig. 1 Characterization of the functionalized TKCP and TKCP@DEX. TEM images of TKCP (a) and TKCP@DEX (b) NPs. Scale bar: $200 \mathrm{~nm}$. c DLS characterization of TKCP and TKCP@DEX NPs. d The zeta potential of TKCP and TKCP@DEX NPs. e UV-vis absorption spectra of Cy5.5, BHQ-3 and TKCP NPs. Fluorescence intensity $(\mathbf{f})$ and Relative fluorescence intensity $(\mathbf{g})$ of TKCP in different concentrations of $\mathrm{KO}_{2}(0 \mu \mathrm{M}, 50 \mu \mathrm{M}$ and $100 \mu \mathrm{M})$ or ROS inhibitor (NAC) for different times. Ex/Em of Cy5.5: $675 / 695 \mathrm{~nm}$. $\mathbf{h}$ Size change of the TKCP NPs incubated in pH 7.4 PBS buffer containing $\mathrm{KO}_{2}(0,50$ and $100 \mu \mathrm{M})$ or with ROS inhibitor (NAC) for different times. i Cumulative DEX release from the TKCP@DEX NPs after incubation in PBS containing different concentrations of $\mathrm{KO}_{2}(0 \mu \mathrm{M}, 50 \mu \mathrm{M}$ and $100 \mu \mathrm{M})$ or with ROS inhibitor (NAC) for different times at $37^{\circ} \mathrm{C}$. $(n=3$, mean $\pm S D$ )

suggesting that the disassembly of TKCP NPs was triggered in response to oxidative milieu (Fig. 1h).

\section{In vitro drug release at ROS-simulated levels}

As confirmed by the results of in vitro fluorescent recovery study (Fig. 1g), the high level of ROS in OA intracellular could break thioketal linkers and also trigger the release of DEX. The drug loading and embedding ratio were 13\% and 30\% for the TKCP@DEX NPs, respectively. The in vitro drug release showed that only $19 \%$ drug was released in the absence of $\mathrm{KO}_{2}$ (ROS inducer) after incubation for $48 \mathrm{~h}$ (Fig. 1i). The drug release reached up to $42 \%$ and $58 \%$ after treatment with $50 \mu \mathrm{M}$ and $100 \mu \mathrm{M}$ $\mathrm{KO}_{2}$ for $48 \mathrm{~h}$, respectively, indicating obviously accelerated DEX release from the TKCP@DEX NPs by $\mathrm{KO}_{2}$ in a dose-dependent manner. The drug release could be inhibited by the ROS inhibitor NAC (only $26 \%$ of DEX release), because NAC could significantly prevent the production of ROS and the degradation of thioketal linkers. All these results confirmed that DEX released slowly in PBS, showing no notable change without $\mathrm{KO}_{2}$. On the contrary, DEX rapidly increased in the presence of $\mathrm{KO}_{2}$, suggesting that the disassembly of TKCP NPs was triggered by ROS according to the concentration of $\mathrm{KO}_{2}$.

\section{Cytotoxicity analysis}

The cytotoxicity of TKCP and TKCP@DEX NPs on chondrocytes was evaluated by CCK- 8 assay. As show in Fig. 2a, no toxicity was observed with TKCP at the range of 0 to $200 \mu \mathrm{g} / \mathrm{mL}$ after $24 \mathrm{~h}$ and $48 \mathrm{~h}$ incubation. After DEX loading, $25 \mu \mathrm{g} / \mathrm{mL}$ TKCP@DEX (equivalent concentration of DEX was $3 \mu \mathrm{g} / \mathrm{mL}$ ) promoted cell growth compared with untreated chondrocytes (Fig. 2b). Thus, $25 \mu \mathrm{g} / \mathrm{mL}$ TKCP@DEX was selected for further study.

The effect of TKCP@DEX on the cell viability of MIA (ROS inducer)-induced chondrocytes was investigated by CCK-8 analysis. As shown in Fig. 2c, the cell viability 
of the chondrocytes treated with MIA decreased 40.7\% compared with the normal cells. However, after treating with DEX, CAPP@DEX, TKP@DEX and TKCP@ DEX for $24 \mathrm{~h}$, the cell viability was increased to $70.27 \%$, $74.14 \%, 78.78 \%$ and $87.97 \%$ compared with the MIA group, respectively, suggesting potential function of protecting chondrocytes from catabolic activity of inflammatory factor and promoting chondrocytes proliferation.

\section{Hemolysis test}

The biocompatibility of TKCP was measured by the hemolysis test. As shown in Fig. 2d, the TKCP at various concentrations induced lower than $4.0 \%$ hemolysis rate by contacting erythrocytes at $37^{\circ} \mathrm{C}$ for $1 \mathrm{~h}$. These results indicated that TKCP NPs were considered as excellent biocompatible and nonhemolytic materials, showing a great potential of biomaterials for clinical applications.

\section{Cell uptake and in situ fluorescent release}

To investigate the chondrocytes targeting property of TKCP NPs, cellular uptake of TKCP and TKP (without chondrocyte-affinity CAP peptide) NPs were compared. Treatment with $3 \mu \mathrm{M}$ and $6 \mu \mathrm{M}$ MIA caused a 1.1-fold and 2.7 -fold increase of red fluorescence $(46.40 \%$ and $82.51 \%)$ in the cellular uptake compared with untreated cells (22.19\%), respectively (Fig. 2e and f), which revealed that thioketal linkages exhibited efficient ROS-dependent degradation. The fluorescence intensity of TKCP group was observed to be much stronger than that of TKP group after treatment with $3 \mu \mathrm{M}$ MIA (Fig. 2e and g). However, the fluorescence intensity declined to $30.55 \%$ after treating with NAC, which was similar to that of normal cell environment due to the elimination of ROS (Fig. 2e and f). These results indicated that ROS could trigger the cleavage of thioketal linkages to release Cy5.5 from TKCP according to the level of ROS at simulated pathological conditions, and the targeting effect of CAP moieties in TKCP NPs could facilitate high affinity to ECM-rich chondrocytes compared those with non-targeting modality. But in normal condition, Cy5.5 was hardly released and activated. The flow cytometer was used to monitor the ROS production in chondrocytes (Fig. 2h). At OA microenvironment induced by MIA (the concentration was $3 \mu \mathrm{M}$ ), an elevated level of
ROS (29.38\%) was produced compared with normal cells $(12.75 \%)$. And the ROS was up to $42.76 \%$ at the concentration of $6 \mu \mathrm{M}$ MIA, indicating a significant increase of ROS production dependent on the concentration of MIA. Furthermore, the high level of ROS could decrease down to $19.63 \%$ after treated with NAC. Thus, TKCP may potentiate smart real-time monitoring of ROS levels.

\section{In vitro anti-inflammatory activity}

We then evaluated the efficacy of treatment groups to induce crucial OA catabolic biomarkers $(M M P-13, I L-6$, and $M M P-3)$ and chondrogenic markers genes (Col2a1) by qRT-PCR (Fig. 3a). It showed that the $M M P-13, I L-6$ and $M M P-3$ expression of TKCP@DEX group was significantly decreased than that of the MIA-treated group, down to $80.85 \%, 91.30 \%$ and $64.19 \%$, respectively. Compared with DEX, there were $43.47 \%, 23.37 \%$ and $14.19 \%$ decreases of MMP13, IL-6 and MMP-3 expression in TKCP@DEX group respectively, indicating its pronounced anti-inflammatory effect. TKCP@DEX group also significantly up-regulated the expression of Col2a1, one key component of the cartilage matrix compared with other groups after $24 \mathrm{~h}$ of culture (Fig. 3a), suggesting that TKCP@DEX NPs inhibited the degradation of collagen II and protected chondrocytes under the pathological conditions of OA. The expression of OA biomarkers by immunofluorescent staining further confirmed the PCR results (Fig. 3b and c). The results showed the expressions of MMP-13 and IL- 6 were both decreased in the TKCP@DEX group, almost 25-45\% lower than other groups (MIA, DEX, CAPP@DEX and TKP@DEX groups).

\section{In vivo ROS-responsive activity}

To detect the ROS activity at the early stage of OA, we established the OA model by IA injection of MIA (ROS inducer). In the normal group (healthy joints) treatment, IA injection of TKCP probes resulted in weak fluorescent signal in the normal group (healthy joints) and the relative fluorescence intensity was 1.20 at 1 day (Fig. 4a and b). By contrast, a strong fluorescent signal was detected in the OA joints, indicating significantly up-regulated ROS activity in the OA joints compared to healthy joints. The relative fluorescence intensity of TKCP group in OA

(See figure on next page.)

Fig. 2 In vitro cellular evaluation. Cell cytotoxicity of TKCP against chondrocytes (a) and the effect of TKCP@DEX on chondrocytes (b) determined by the CCK-8 assay after incubating for 24 or 48 h. c Cell viability of MIA-induced chondrocytes pretreated with culture medium only, DEX, CAPP@ DEX,TKP@DEX orTKCP@DEX for 24 h.d The hemolysis ratio of TKCP@DEX at different concentrations. e The cellular uptake of TKCP, TKP or CAPP NPs in normal chondrocytes, MIA-induced OA chondrocytes and in the presence of ROS inhibitor (NAC) for $24 \mathrm{~h}$. The nuclei were counterstained with DAPI (blue). $\mathbf{f}$ Quantification of fluorescence after incubation with TKCP for different concentrations of MIA ( $3 \mu \mathrm{M}$ and $6 \mu \mathrm{M})$ or with ROS inhibitor (NAC) for $24 \mathrm{~h} . \mathbf{g}$ Quantification of fluorescence after incubation with TKCP, TKP or CAPP NPs in the presence of MIA (3 $\mu$ M) for $24 \mathrm{~h}$. Flow cytometry analysis (h) the production of intracellular ROS in chondrocytes at different concentrations of MIA ( $3 \mu \mathrm{M}$ and $6 \mu \mathrm{M})$ or with ROS inhibitor (NAC) after incubation for 24 h. Scale bars: $100 \mu \mathrm{m}$. ( $n=3$; mean \pm SD; ${ }^{*}$, \# indicate $p<0.05$; **, \#\# indicate $p<0.01$; **, \#\#\# indicate $p<0.001$.) 


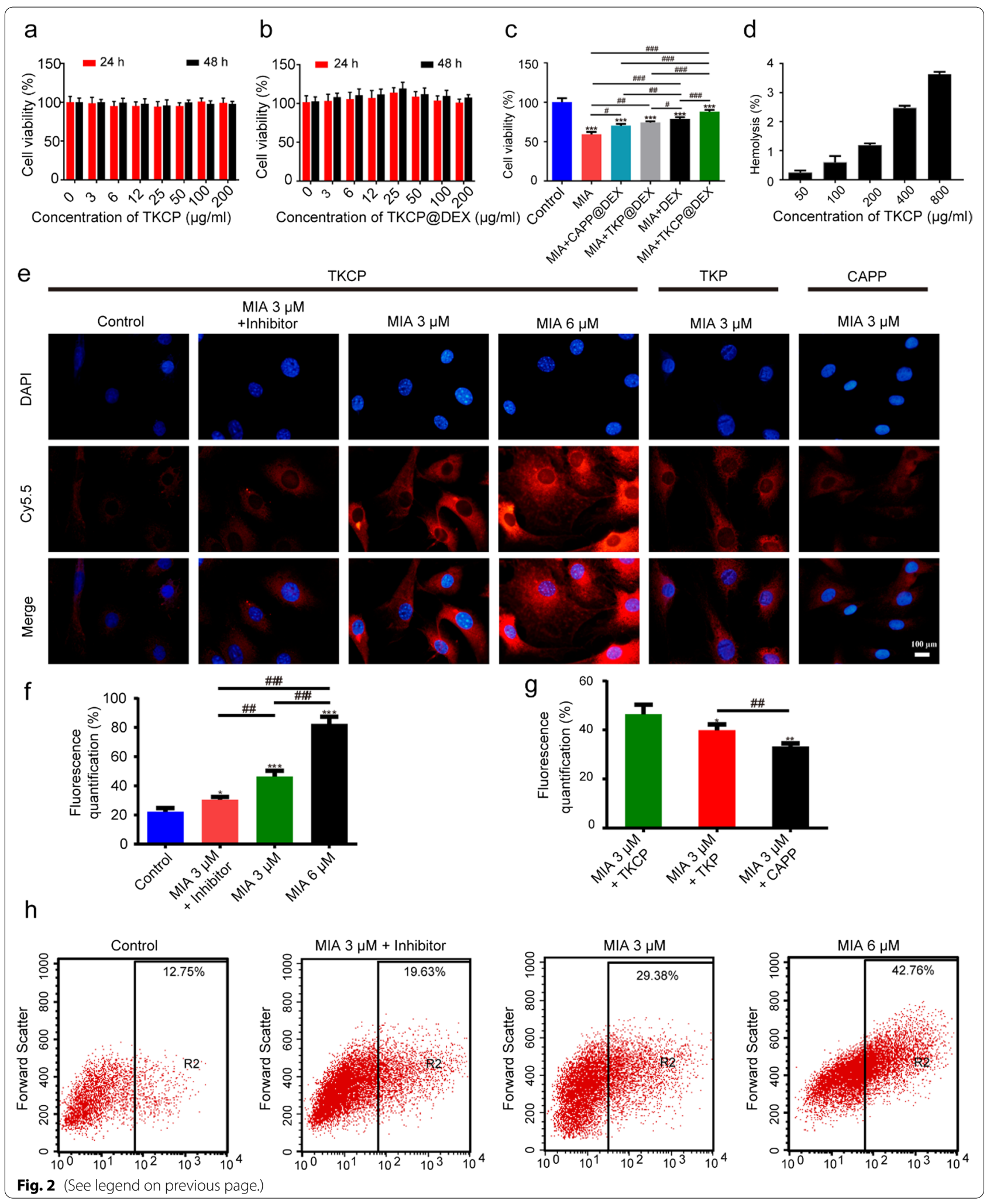




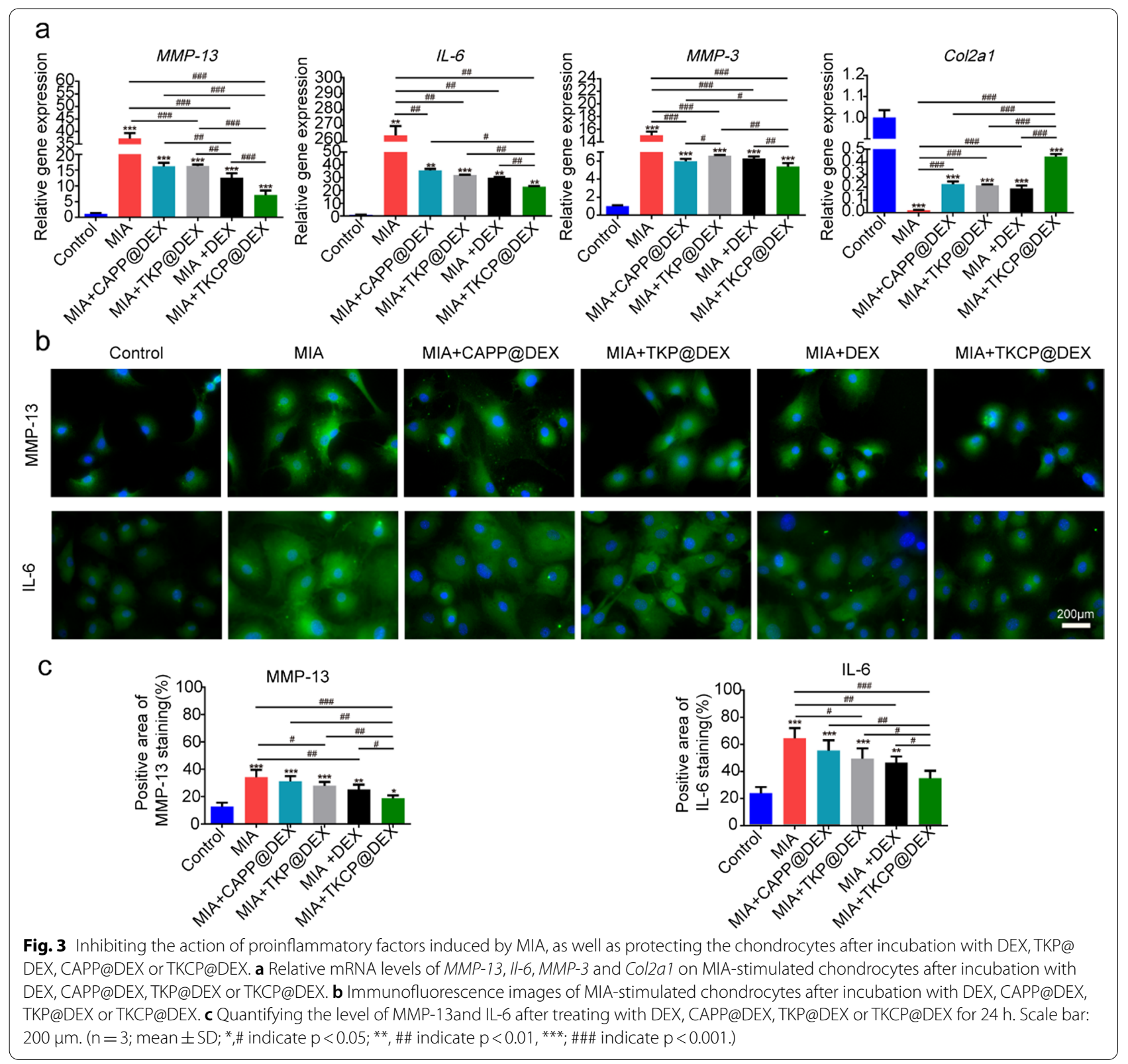

sites reached peak intensity at 1 day, and still maintained obvious fluorescence at 14 days. Moreover, the relative fluorescence intensity of TKCP NPs treated mice was nearly $\sim 1$.34-fold higher than that of CAPP NPs, and $\sim 0.95$-fold higher than TKP NPs (Fig. 4a and b) at 7 days, indicating that the decoration of CAPP endowed the potential enrichment effect of TKCP NPs and thioketal linkage could be effectively cut off by ROS in cartilage. Thus, TKCP NPs exhibited cartilage targeting property and prolonged retention time in joints, which was suitable for effective bioimaging to detect the development of OA in vivo.
Compared with the OA mice (0.05 mg MIA) injected with the TKCP probes, those injected with the TKCP + ROS inhibitor group produced weaker fluorescence signal (Fig. $4 \mathrm{c}$ and $\mathrm{d}$ ). In addition, when mice injected with $0.1 \mathrm{mg}$ MIA, a higher ROS circumstance was induced, leading to more serious joint damage (Fig. 4c and d). And the relative fluorescence intensities could be increased at higher ROS circumstance (IA with $0.1 \mathrm{mg}$ MIA), increasing up to $30 \%$ higher than that of IA with $0.05 \mathrm{mg}$ MIA after treated with TKCP probes. This result indicated that the intensity of fluorescent signals of TKCP probe could respond to 


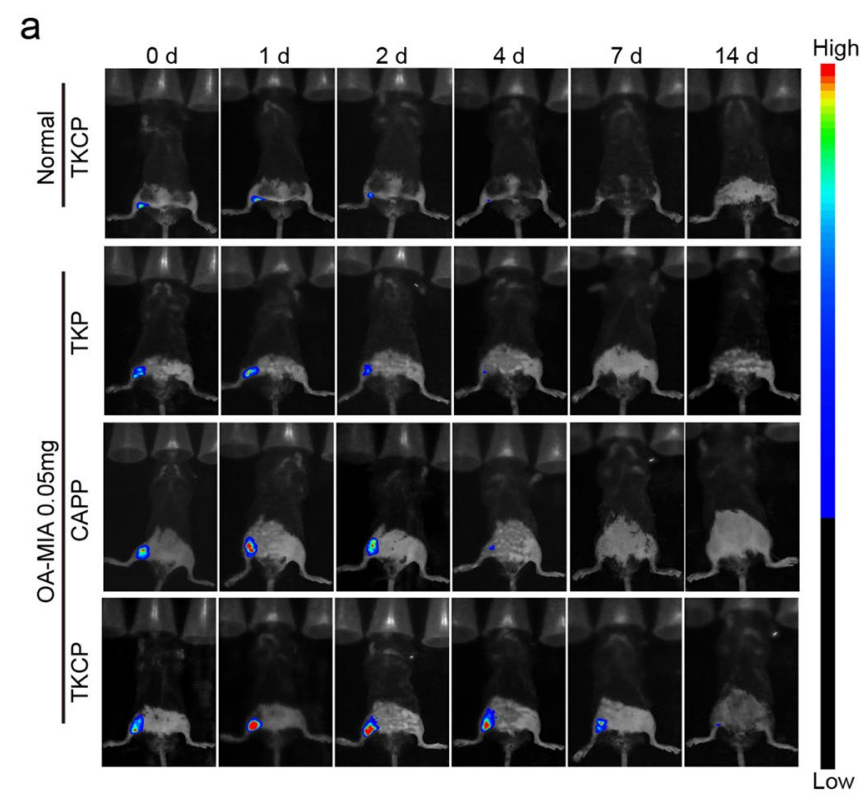

b

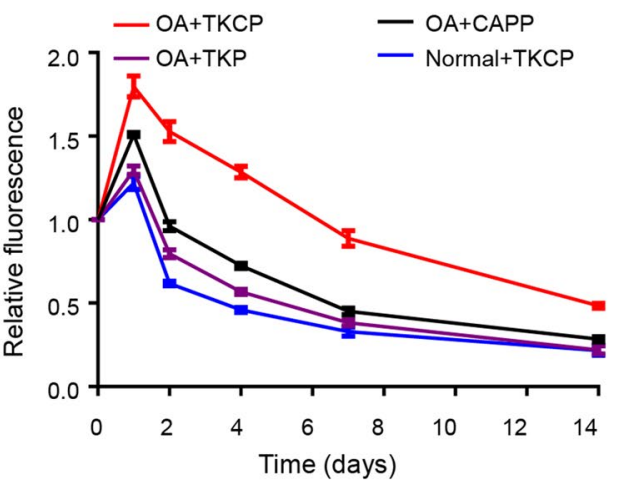

C

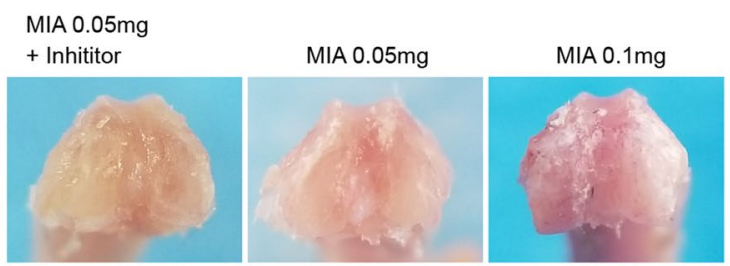

d
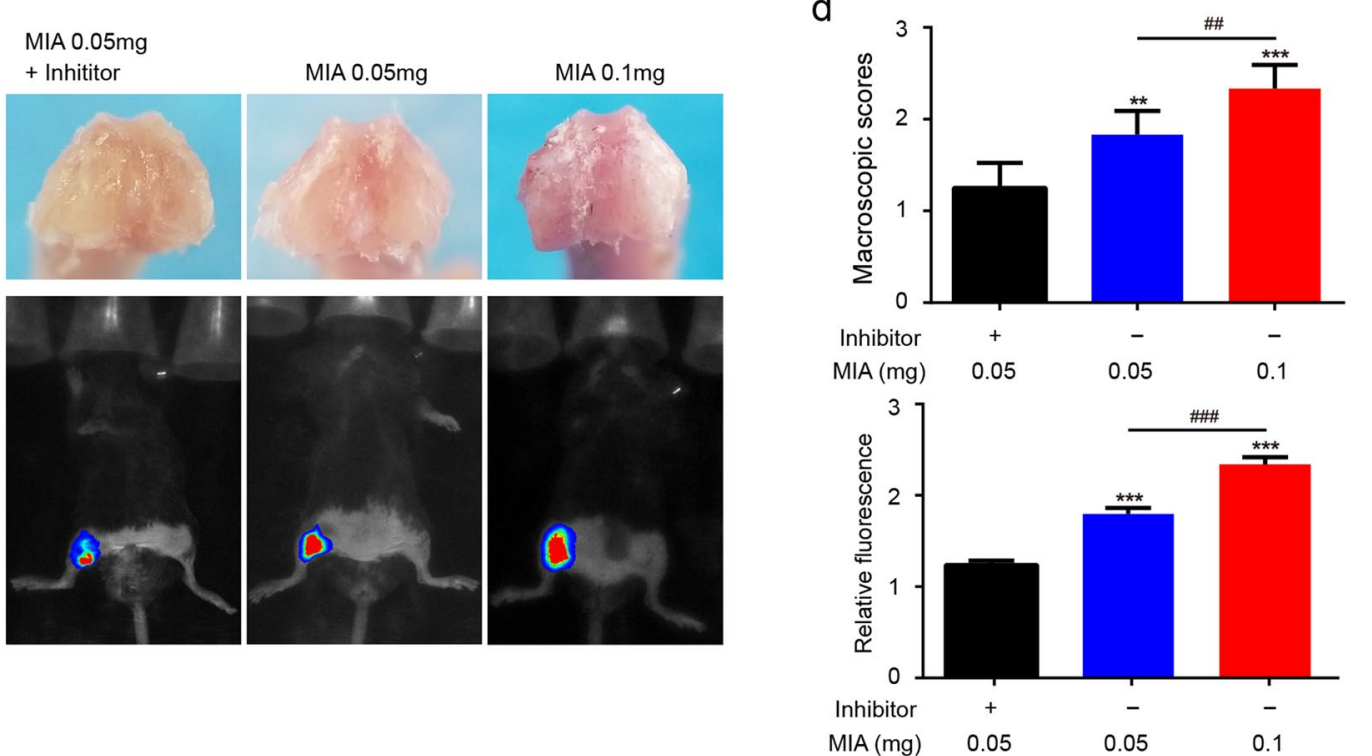

Fig. 4 In vivo fluorescent image after IA injection of CAPP, TKP or TKCP probes to detect the level of ROS induced by MIA in C57BL6/J knees. a The up-regulated ROS activity in the OA joints was reflected by fluorescent signal and measured by an in vivo imaging system at selected time points after IA-injection. Ex: $630 \mathrm{~nm}, \mathrm{Em}: 700 \mathrm{~nm}$. b The relative fluorescence intensity of TKCP, CAPP or TKP probes at different treatment times. $\mathbf{c}$ Representative photographs showed the macroscopic appearance of the cartilage from the femoral condyles and in vivo NIR bioimaging indicated the level of ROS via the fluorescent intension according to the development of OA. $\mathbf{d}$ Analysis of the macroscopic score and relative fluorescent intensity to evaluate the severity of OA. $\left(n=3\right.$; ean $\pm S D ;{ }^{*}$, \# indicate $p<0.05,{ }^{*}$; \#\# indicate $p<0.01$; **, \#\#\# indicate $p<0.001$.)

ROS related OA severity, favorable for precise disease classification.

\section{In vivo treatment effect}

We further carried out in vivo experiments to evaluate the OA therapeutic efficacy of TKCP@DEX. The mice were divided into six groups, including PBS, OA, DEX,
CAPP@DEX, TKP@DEX and TKCP@DEX. In the PBStreated group, general characteristic OA features, such as surface irregular and large cartilage erosion, were observed. The macroscopic score of OA group was significantly higher than that of control group over time, which indicated mild or moderate cartilage destruction based on pathological alteration of joint morphology from 

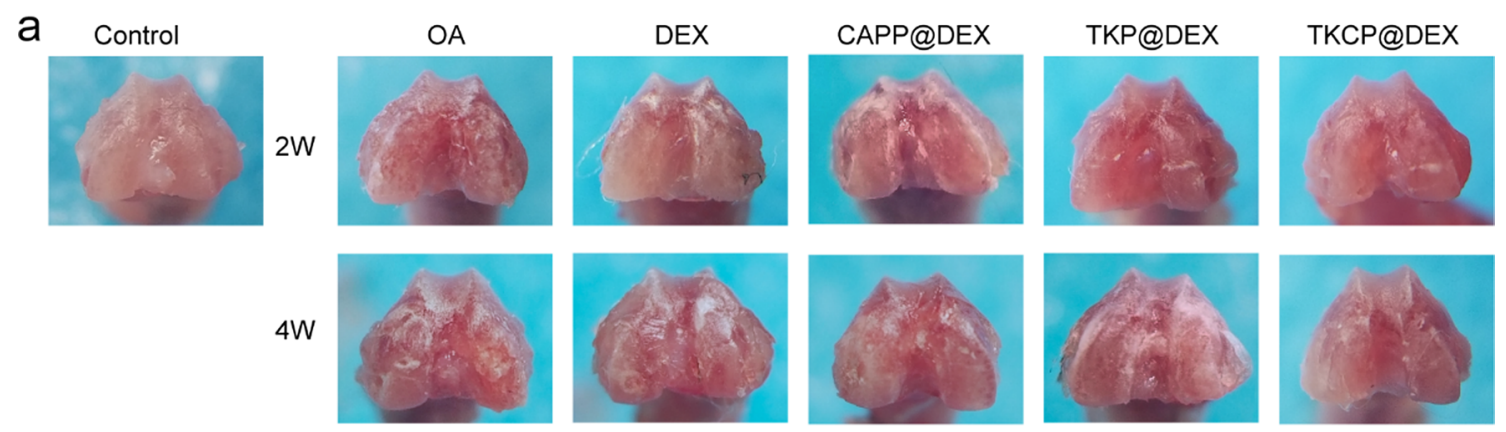

b
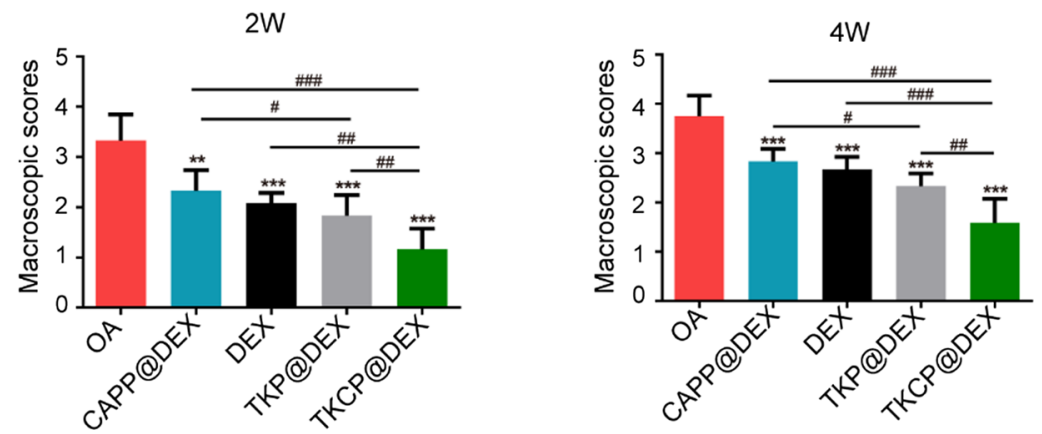

Fig. 5 Macroscopic appearance and scoring of cartilage femoral condyles after treatment for 2 and 4 weeks. a The macroscopic observation and $\mathbf{b}$ the macroscopic scores of cartilages after IA-injection with PBS, DEX, CAPP@DEX,TKP@DEX or TKCP@DEX ( $n=6$; mean \pm SD)

2 to 4 weeks (Fig. 5a). After treatment with DEX, there was a $37.50 \%$ and $28.89 \%$ reduction in the joint score in 2 weeks and 4 weeks, respectively, as compared to that of treatment with PBS alone. Meanwhile, both CAPP@DEX and TKP@DEX treatment significantly decreased the severity of OA. Furthermore, there was a $65 \%$ and $57.78 \%$ reduction in TKCP@DEX group after treatment for 2 and 4 weeks, respectively, compared to that OA group, which showed its effective therapeutic repair effect of OA (Fig. 5b).

The $\mathrm{HE}$ and safranin $\mathrm{O} /$ fast green staining were used to evaluate the therapy efficacy of TKCP@DEX in vivo (Fig. 6a and b). In the OA group, OA characteristics, including cartilage damage, fibrillated lesions and loss of aggrecan worsened over time, were showed in the progression of OA. In contrast, TKCP@DEX treatment resulted in a significant improvement in matrix arrangement, tide line maintenance or cartilage lesion, and the OARSI score exhibited improvement, with a reduce of $86.7 \%$ and $83 \%$ compared with OA group at 2 and 4 weeks, respectively (Fig. 6c). The scores for the TKCP@ DEX group were markedly lower than that of other treatment groups, exhibiting positive effects on the restoration of cartilage matrix expression.

We also assessed the expression of MMP-13 in OA cartilage by immunohistochemistrical analysis, which is a degradation product of type II collagen and corresponds to articular cartilage damage in the early stages of OA. The positive staining of MMP-13 could hardly be detected in the normal cartilage. In contrast, it showed high expression in the superficial and middle zones of the cartilage in $\mathrm{OA}$ group. Following treatment with DEX, CAPP@DEX, TKP@DEX or TKCP@DEX, osteoarthritic changes of MMP-13 over-expression was reduced (Fig. 6d). These results indicated that TKCP@DEX significantly slowed the progression of early OA and prevented the severe damage to articular cartilage in the OA.

\section{Discussion}

The abnormal over-production of endogenous ROS within cartilage tissue is a key hallmark of OA [48], providing a disease-specific triggering mechanism for drug control and release systems. Herein, we fabricated an advance ROS responsive and cartilage targeting TKCP@ DEX nanoprobe with loaded drug for imaging and effective therapy of OA, which may provide reference for clinical application.

As a promising domain, stimuli responsive theranostic nanoprobes have been widely applied in disease theranostic owing to their unique all-in-one features [49-51]. Several researchers proposed ultrasensitive ROS-responsive carriers that contained agents to release the drug to the disease site $[48,52,53]$. Compared with unresponsive delivery systems, these ROS-responsive systems remain stable in normal tissue, preventing their release to noninflamed tissues, which suggested their specificity in 


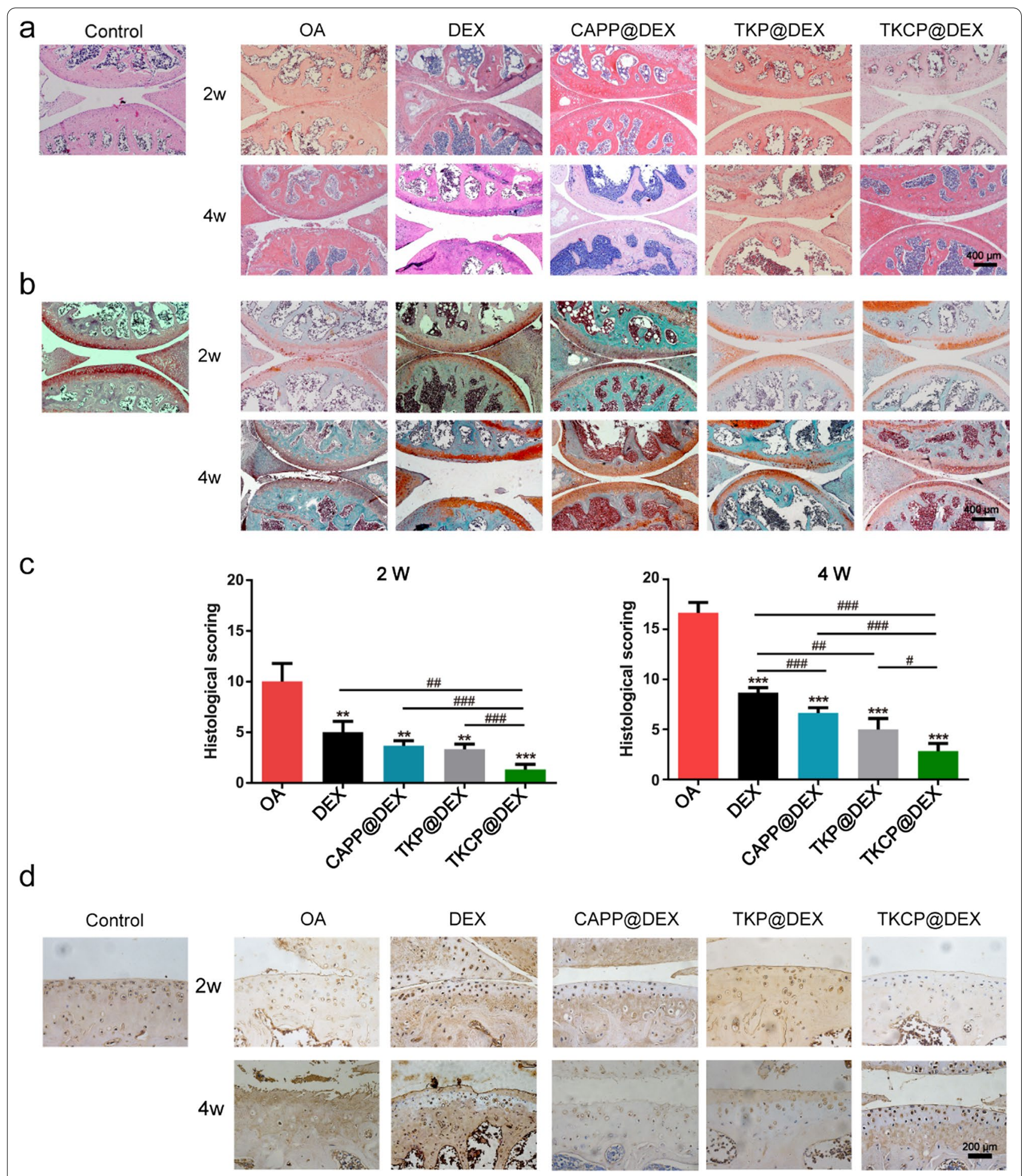

Fig. 6 Histological evaluations of cartilage femoral condyles after IA injection of probes for 2 weeks and 4 weeks. H\&E (a) and safranin-O/fast green (b) staining of cartilage sections. Scale bar: $400 \mu \mathrm{m}$. c Histological scoring of articular cartilage. d Immunohistochemical staining of MMP-13 was evaluated on cartilage sections after IA-injection with PBS, DEX, CAPP@DEX, TKP@DEX or TKCP@DEX. Scale bar: $200 \mu m$. $(n=6 ;$ mean \pm SD; * , \# indicate $p<0.05,{ }^{* *}$; \#\# indicate $p<0.01 ;{ }^{* * *}$, \#\#\# indicate $p<0.001$.) 
inflammation site. But these ROS responsive nano systems can only control the release of drugs, they cannot visually real-time monitor the progression of disease and most of them have no therapeutic effect. In our study, TKCP is superior to unresponsive CAPP, as evidenced by fluorescent recovery studies simulated by abundant of ROS in vitro and vivo (Figs. 1f, g, 2e and 4a). The fluorescent signal is strong in the OA microenvironment, but is extremely weak in normal chondrocytes and joints, indicating that the level of fluorescence signal correlates with ROS content. TKCP@DEX not only exhibits smart drug release potential in response to high levels of ROS as demonstrated by drug release behavior test (Fig. 1i), but also can real-time track ROS activity since it emits signal of fluorescence varied with ROS, promising for monitoring and on-demand therapy of OA.

On the other hand, cartilage targeting is of importance for drug therapy. Most in situ injected particles may be quickly cleared by the joint fluid because of the inability of homing to lesion sites, leading to application limitations in OA diagnosis and treatment. Pi et al. engineered a chondrocyte-affinity peptide (CAP, DWRVIIPPRPSA) [32] by phage display technology. In our study, the nanoprobe TKCP with cartilage targeting CAP showed stronger fluorescence than TKP (without CAP), as observed in vitro cellular uptake tests (Fig. 2e) and in vivo NIR imaging (Fig. 4). Moreover, the CAP-modified TKCP@DEX prolonged the retention time of nanoparticles in the joint (Fig. 4a and b), which could improve therapeutic outcome of OA. These results indicated that cartilage targeting ensured the real-time monitoring and effective treatment of $\mathrm{OA}$.

In our studies, we observed that the TKCP@DEX NPs showed stronger therapeutic efficacy than that of delivery systems without conjugation of CAP and TK. The TKCP@DEX NPs play a key role in relieving the inflammation in vitro and in vivo (Figs. 3, 5, 6). The results of macroscopic and histological analysis further evaluated that IA injection of TKCP@DEX showed the better therapeutic effect than other formulations (TKP@ DEX, CAPP@DEX or free DEX) after 2 and 4 weeks of treatment (Figs. 5, 6). In addition, hemolysis test further assessed the excellent biocompatibility of the TKCP@ DEX NPs platform (Fig. 2d). The above results revealed that the drug delivery system targeted to the cartilage and responded well to low concentrations of ROS, exhibiting highly sensitive imaging and effective anti-inflammatory activity for therapy of OA.

\section{Conclusions}

In summary, we have successfully fabricated a novel cartilage-targeting and ROS-responsive delivery platform for imaging and therapy of OA, which enables real-time imaging to monitor the severity of $\mathrm{OA}$ and on-demand drug release at the site of abnormal ROS milieu. We expect that this theranostic system may be applied in clinic for treatment of OA.

\section{Supplementary Information}

The online version contains supplementary material available at https://doi. org/10.1186/s12951-021-01136-4.

Additional file 1. All data generated or analysed during this study are included in this published article and its additional files.

\section{Acknowledgements}

This study was financially supported by National Natural Science Fundation of China (Grant No.81960410, 81960414), the Guangxi Key Research and Development Plan (Grant No. GuikeAD19254003), the Guangxi Science and Technology Major Project (Grant No. GuikeAA19254002). We really appreciated for their kindly help with the In-vivo optical imaging by the Center for Translational Medicine of Guangxi Medical University (Nanning, China).

\section{Authors' contributions}

$C S, M G, H M C, Z N Q$ and $L Z$ designed experiments and wrote the manuscript. CS, MG, and HMC synthesized and analyzed the nanodrugs. CS, MG, and HMC conducted the vitro and vivo experiments. CS, MG and HMC conducted the in vivo optical imaging and analysis the results. JMZ and LZ provided the fund for this research. QML, ZML and WX helped to edit the figures. YTZ performed statistical analysis of the data. All authors read and approved the final manuscript.

\section{Declarations}

Ethics approval and consent to participate

Animal care conformed to institutional guidelines. All animal studies were agreed by the Institutional Ethics Committee of Guangxi Medical University.

\section{Consent for publication}

All authors agree to be published.

\section{Competing interests}

No potential conflicts of interest were disclosed.

\section{Author details}

${ }^{1}$ Guangxi Engineering Center in Biomedical Materials for Tissue and Organ Regeneration, The First Affiliated Hospital of Guangxi Medical University, Nanning 530021, China. ${ }^{2}$ Department of Orthopedics, The Affiliated Hospital of Guilin Medical University, No. 15 Lequn Road, Guilin 541001, Guangxi, China. ${ }^{3}$ Guangxi Collaborative Innovation Center for Biomedicine, The First Affiliated Hospital of Guangxi Medical University, Nanning 530021, China. ${ }^{4}$ Department of Orthopaedics Trauma and Hand Surgery, The First Affiliated Hospital of Guangxi Medical University, Nanning 530021, China. ${ }^{5}$ Guangxi Key Laboratory of Regenerative Medicine, International Joint Laboratory On Regeneration of Bone and Soft Tissue, The First Affiliated Hospital of Guangxi Medical University, Nanning 530021, China.

Received: 17 June 2021 Accepted: 14 November 2021

Published online: 27 November 2021

\section{References}

1. Cross M, Smith E, Hoy D, Nolte S, Ackerman I, Fransen M, Bridgett L, Williams S, Guillemin F, Hill CL, Laslett LL, Jones G, Cicuttini F, Osborne R, Vos T, Buchbinder R, Woolf A, March L. The global burden of hip and knee osteoarthritis: estimates from the global burden. Ann Rheum Dis. 2014;73:1323-30. 
2. Holt HL, Katz JN, Reichmann WM, Gerlovin H, Wright EA, Hunter DJ, Jordan JM, Kessler CL, Losina E. Forecasting the burden of advanced knee osteoarthritis over a 10-year period in a cohort of 60-64 year-old US adults. Osteoarthr Cartil. 2011;19:44-50.

3. Bijlsma JWJ, Berenbaum F, Lafeber FPJG. Osteoarthritis: an update with relevance for clinical practice. Lancet. 2011;377:2115-26.

4. Jin P, Wiraja C, Zhao JM, Zhang JL, Zheng L, Chenjie Xu CJ. Nitric oxide nanosensors for predicting the development of osteoarthritis in rat. ACS Appl Mater Interfaces. 2017:9:25128-37.

5. Lee S, Park K, Lee SY, Ryu JH, Park JW, Ahn HJ, Kwon IC, Youn IC, Kim K, Choi K. Dark quenched matrix metalloproteinase fluorogenic probe for imaging osteoarthritis. Bioconjug Chem. 2008;19:1743-7.

6. Castano AD, Lim NH, Tranchant I, Amoura M, Beau F, Wieland H, Kingler O, Herrmann M, Nazaré M, Plettenburg O, Dive V, Vicent MJ, Nagase H. In vivo imaging of MMP-13 activity using a specific polymer-FRET peptide conjugate detects early osteoarthritis and inhibitor efficacy. Adv Funct Mater. 2018;28:37.

7. Lim NH, Meinjohanns E, Meldal M, Gharios GB, Nagase H. In vivo imaging of MMP-13 activity in the murine destabilised medial meniscus. Osteoarthr Cartil. 2014;22:862-8.

8. Ryu JH, Lee A, Lee S, Ahn CH, Park JW, Leary JF, Park S, Kim K, Kwon IC, Youn I-C, Choi K. "One-step" detection of matrix metalloproteinase activity using a fluorogenic peptide probe-immobilized diagnostic kit. Bioconjug Chem. 2010:21:1378-84

9. Bellucci F, Meini S, Cucchi P, Catalani C, Nizzardo A, Riva A, Guidelli GM, Ferrata P, Fioravanti A, Maggi CA. Synovial fluid levels of bradykinin correlate with biochemical markers for cartilage. Osteoarthr Cartil. 2013;21:1774-80.

10. Karan A, Karan MA, Vural P, Erten N, Taşçioğlu C, Aksoy C, Canbaz M, Oncel A. Synovial fluid nitric oxide levels in patients with knee osteoarthritis. Clin Rheumatol. 2003:22(6):397-9.

11. Maher MC, Schnabel LV, Cross JA, Papich MG, Divers TJ, Fortier LA. Plasma and synovial fluid concentration of doxycycline following low-dose, lowfrequency administration, and resultant inhibition of matrix metalloproteinase-13 from interleukin-stimulated equine synoviocytes. Equine Vet J. 2014;46:198-202.

12. Beekman B, El BV, Drijfhout JW, Ronday HK, TeKoppele JM. Highly increased levels of active stromelysin in rheumatoid synovial fluid determined by a selective fluorogenic assay. FEBS Lett. 1997;418:305-9.

13. Lepetsos P, Papavassiliou AG. ROS/oxidative stress signaling in osteoarthritis. Biochim Biophys Acta. 2016;1862:576-91.

14. Yao YJ, Zhang HL, Wang ZY, Ding J, Wang SQ, Huang BQ, Ke SF, Gao CY. Reactive oxygen species (ROS)-responsive biomaterials mediate tissue microenvironments and tissue regeneration. J Mater Chem B. 2019;7:5019-37.

15. Lotito APN, Muscará MN, Kiss MHB, Teixeira SA, Novaes GS, Laurindo IMM, Silva CA, Mello SBV. Nitric oxide-derived species in synovial fluid from patients with juvenile idiopathic arthritis. J Rheumatol. 2004;31:992-7.

16. Hosseinzadeh A, Kamrava SK, Joghataei MT, Darabi R, Zadeh AS, Shahriari M, Reiter RJ, Ghaznavi H, Mehrzadi S. Apoptosis signaling pathways in osteoarthritis and possible protective role of melatonin. J Pineal Res. 2016;61:411-25

17. Yuan YY, Liu J, Liu B. Conjugated-polyelectrolyte-based polyprodrug: targeted and image-guided photodynamic and chemotherapy with ondemand drug release upon irradiation with a single light source. Angew Chem Int Ed Engl. 2014;53:7163-8.

18. Lee S, Stubelius A, Hamelmann N, Tran V, Almutairi A. Inflammationresponsive drug-conjugated dextran nanoparticles enhance anti-inflammatory drug efficacy. ACS Appl Mater Interfaces. 2018;10:40378-87.

19. Kim EJ, Bhuniya S, Lee H, Kim HM, Cheong C, Maiti S, Hong KS, Kim JS. An activatable prodrug for the treatment of metastatic tumors. J Am Chem Soc. 2014;136:13888-94

20. LvW, Xu JP, Wang XQ, Li XR, Xu QW, Xin HL. Bioengineered boronic ester modified dextran polymer nanoparticles as reactive oxygen species responsive nanocarrier for ischemic stroke treatment. ACS Nano. 2018;12:5417-26.

21. Yang $B$, Wang KY, Zhang D, Sun BJ, Ji B, Wei L, Li ZB, Wang ML, Zhang XB, Zhang HT, Kan QM, Luo C, Wang YJ, He ZG, Sun J. Light-activatable dualsource ROS-responsive prodrug nanoplatform for synergistic chemophotodynamic therapy. Biomater Sci. 2018;6:2965-75.
22. Jung E, Noh JY, Kang CS, Yoo D, Song C, Lee D. Ultrasound imaging and on-demand therapy of peripheral arterial diseases using $\mathrm{H}_{2} \mathrm{O}_{2}$-activated bubble generating anti-inflammatory polymer particles. Biomaterials. 2018;179:175-85

23. Kang CS, Cho W, Park M, Kim J, Park S, Shin D, Song C, Lee D. $\mathrm{H}_{2} \mathrm{O}_{2}$-triggered bubble generating antioxidant polymeric nanoparticles as ischemia/reperfusion targeted nanotheranostics. Biomaterials. 2016;85:195-203.

24. Kim S, Park H, Song Y, Hong D, Kim O, Jo E, Khang G, Lee D. Reduction of oxidative stress by p-hydroxybenzyl alcohol-containing biodegradable polyoxalate nanoparticulate antioxidant. Biomaterials. 2011;32:3021-9.

25. Xu XD, Saw PE, Tao W, Li YJ, Ji XY, Bhasin S, Liu YL, Ayyash D, Rasmussen J, Huo M, Shi JJ, Farokhzad OC. ROS-responsive polyprodrug nanoparticles for triggered drug delivery and effective cancer therapy. Adv Mater. 2017:29:201700141

26. Zhang WJ, Hu XL, Shen Q, Xing D. Mitochondria-specific drug release and reactive oxygen species burst induced by polyprodrug nanoreactors can enhance chemotherapy. Nat Commun. 2019;10:1704.

27. Cheng DB, Zhang XH, Yu-Juan Gao YJ, Ji L, Hou DY, Wang ZQ, Xu WH, Qiao $Z Y$, Wang $H$. Endogenous reactive oxygen species-triggered morphology transformation for enhanced cooperative interaction with mitochondria. J Am Chem Soc. 2019;141:7235-9.

28. Kavanaugh TE, Werfel TA, Cho H, Hasty KA, Duvall CL. Particle-based technologies for osteoarthritis detection and therapy. Drug Deliv Transl Res. 2016;6:132-47.

29. Rothenfluh DA, Bermudez H, O'Neil CP, Hubbell JA. Biofunctional polymer nanoparticles for intra-articular targeting and retention in cartilage. Nat Mater. 2008;7:248-54

30. Maeda $\mathrm{H}$, Nakamura $\mathrm{H}$, Fang J. The EPR effect for macromolecular drug delivery to solid tumors: improvement of tumor uptake, lowering of systemic toxicity, and distinct tumor imaging in vivo. Adv Drug Deliv Rev. 2013;65:71-9.

31. Ouyang ZX, Tan TT, Liu CF, Duan J, Wang WC, Guo XN, Zhang Q, Li ZL, Huang QL, Dou PC, Liu T. Targeted delivery of hesperetin to cartilage attenuates osteoarthritis by bimodal imaging with $\mathrm{Gd}_{2}\left(\mathrm{CO}_{3}\right)_{3} @ P D A$ nanoparticles via TLR-2/NF-KB/Akt signaling. Biomaterials. 2019;205:50-63.

32. Pi YB, Zhang $X$, Shi JJ, Zhu JX, Chen WQ, Zhang CG, Gao WW, Zhou CY, Ao YF. Targeted delivery of non-viral vectors to cartilage in vivo using a chondrocyte-homing peptide identified by phage display. Biomaterials. 2011:32:6324-32.

33. Lu YHCS, Evans CH, Grodzinsky AJ. Effects of short-term glucocorticoid treatment on changes in cartilage matrix degradation and chondrocyte gene expression induced by mechanical injury and inflammatory cytokines. Arthritis Res Ther. 2011;13:R142.

34. Asadullah K, Schäcke H, Cato ACB. Dichotomy of glucocorticoid action in the immune system. Trends Immunol. 2002;23:120-2.

35. Huebner KD, Shrive NG, Frank CB. Dexamethasone inhibits inflammation and cartilage damage in a new model of post-traumatic osteoarthritis. $J$ Orthop Res. 2014;32:566-72.

36. Rao ZT, Wang SQ, Wang JQ. Peroxiredoxin 4 inhibits IL-1 $\beta$-induced chondrocyte apoptosis via PI3KJAKT signaling. Biomed Pharmacother. 2017;90:414-20.

37. Santos MV, Rocha LB, Vieira EG, Oliveira AL, Lobo AO, Carvalho MA, Osajima JA, Silva-Filho EC. Development of composite scaffolds based on cerium doped-hydroxyapatite and natural gums-biological and mechanical properties. Materials. 2019;12:2389.

38. Jiang LP, Li LJ, Geng CY, Gong DZ, Jiang LJ, Ishikawa N, Kajima K, Zhong LF. Monosodium iodoacetate induces apoptosis via the mitochondrial pathway involving ROS production and caspase activation in rat chondrocytes in vitro. J Orthop Res. 2013;31:364-9.

39. Morita K, Miyamoto T, Fujita N, Kubota Y, Ito K, Takubo K, Miyamoto K, Ninomiya K, Suzuki T, Iwasaki R, Yagi M, Takaishi H, Toyama Y, Suda T. Reactive oxygen species induce chondrocyte hypertrophy in endochondral ossification. J Exp Med. 2007;204:1613-23.

40. Park C, Jeong JW, Lee DS, Yim MJ, Lee JM, Han MH, Kim S, Kim HS, Kim GY, Park EK, You- Jeon J, Cha HJ, Choi HY. Sargassum serratifolium extract attenuates interleukin-1 $\beta$-induced oxidative stress and inflammatory response in chondrocytes by suppressing the activation of NF-KB, p38 MAPK, and PI3K/Akt. Int J Mol Sci. 2018;19:2308.

41. Uchimura T, Nakamura DS, Link EM, Noguchi Y, Ōmura S, Sunazuka T, Greenblatt DJ, Zeng L. Erythromycin acts through the ghrelin receptor to 
attenuate inflammatory responses in chondrocytes and maintain joint integrity. Biochem Pharmacol. 2019;165:79-90.

42. Bowles RD, Mata BA, Bell RD, Mwangi TK, Huebner JL, Kraus VB, Setton LA. In vivo luminescence imaging of NF-KB activity and serum cytokine levels predict pain sensitivities in a rodent model of osteoarthritis. Arthritis Rheumatol. 2014;66:637-46.

43. Yamada EF, Salqueiro AF, Goulart AS, Mendes VP, Anjos BL, Folmer V, Silva $M D$. Evaluation of monosodium iodoacetate dosage to induce knee osteoarthritis: relation with oxidative stress and pain. Int J Rheum Dis. 2019;22:399-410

44. Teng P, Liu Y, Dai Y, Zhang HJ, Liu WT, Hu J. Nicotine attenuates osteoarthritis pain and matrix metalloproteinase-9 expression via the a7 nicotinic acetylcholine receptor. J Immunol. 2019;203(2):485-92.

45. Shetty YC, Patil AE, Jalgaonkar SV, Rege NN, Salgaonkar S, Teltumbde PA, Kshirsagar S, Paresh Koli G, Brahma S. Intra-articular injections of ketamine and $25 \%$ dextrose improve clinical and pathological outcomes in the monosodium iodoacetate model of osteoarthritis. J Basic Clin Physiol Pharmacol. 2017;28:543-53.

46. Guingamp C, Pottie PG, Philippe L, Terlain B, Netter P, Gillet P. Monoiodoacetate-induced experimental osteoarthritis: a dose-response study of loss of mobility, morphology, and biochemistry. Arthritis Rheum. 1997:40:1670-9.

47. Pritzker KPH, Gay S, Jimenez SA, Ostergaard K, Pelletier JP, Revell PA, Salter D, Berg WB. Osteoarthritis cartilage histopathology: grading and staging. Osteoarthr Cartil. 2006;14:13-29.

48. Chung MF, Chia WT, Wan WL, Lin YJ, Sung HW. Controlled release of an anti-inflammatory drug using an ultrasensitive ROS-responsive gas-generating carrier for localized inflammation inhibition. J Am Chem Soc. 2015;137:12462-5.

49. Kaliamurthi S, Korkmaz AD, Selvaraj G, Polat EG, Wei YK, Almessiere MA, Baykal A, Gu K, Wei DQ. Viewing the emphasis on state-of-the-art magnetic nanoparticles: synthesis, physical properties, and applications in cancer theranostics. Curr Pharm Des. 2019;25:1505-23.

50. Wan SS, Cheng Q, Zeng X, Zhang XZ. A Mn(III)-sealed metal-organic framework nanosystem for redox-unlocked tumor theranostics. ACS Nano. 2019:13:6561-71.

51. Dai YD, Sun XY, Sun W, Yang JB, Liu R, Luo Y, Zhang T, Tian Y, Lu ZL, He L. $\mathrm{H}_{2} \mathrm{O}_{2}$-responsive polymeric micelles with a benzil moiety for efficient DOX delivery and AIE imaging. Org Biomol Chem. 2019;17:5570-7.

52. Wilson DS, Dalmasso G, Wang LX, Sitaraman SV, Merlin D, Murthy N. Orally delivered thioketal nanoparticles loaded with TNF-a-siRNA target inflammation and inhibit gene expression in the intestines. Nat Mater. 2010;9:923-8.

53. Chen DQ, Zhang GQ, Li RM, Guan MR, Wang XY, Zou TJ, Zhang Y, Wang CR, Shu CY, Hong H, Wan LJ. Biodegradable, hydrogen peroxide, and glutathione dual responsive nanoparticles for potential programmable paclitaxel release. J Am Chem Soc. 2018;140:7373-6.

\section{Publisher's Note}

Springer Nature remains neutral with regard to jurisdictional claims in published maps and institutional affiliations.
Ready to submit your research? Choose BMC and benefit from:

- fast, convenient online submission

- thorough peer review by experienced researchers in your field

- rapid publication on acceptance

- support for research data, including large and complex data types

- gold Open Access which fosters wider collaboration and increased citations

- maximum visibility for your research: over $100 \mathrm{M}$ website views per year

At BMC, research is always in progress.

Learn more biomedcentral.com/submissions 